\title{
ADIÇÃO DE HIDROXITOLUENO BUTILADO (BHT) NO DILUIDOR ACP- 106c PARA CONGELAÇÃO DE SÊMEN CANINO
}

\section{ADDITION OF BUTYLATED HYDROXY TOLUENE (BHT) TO THE ACP-106c EXTENDER FOR CANINE SEMEN FREEZING}

\author{
Herlon Victor Rodrigues Silva ${ }^{1}$ \\ Thalles Gothardo Pereira Nunes ${ }^{1}$ \\ Antônio Cavalcante Mota Filho ${ }^{2}$ \\ José Nicodemos Pinto ${ }^{3}$ \\ Alexandre Rodrigues Silva ${ }^{4}$ \\ Lúcia Daniel Machado da Silva ${ }^{1 *}$ \\ ${ }^{1}$ Universidade Estadual do Ceará, Fortaleza, CE, Brasil. \\ ${ }^{2}$ Faculdades Terra-Nordeste, Caucaia, CE, Brasil. \\ ${ }^{3}$ Médico Veterinário Autônomo, Fortaleza, CE, Brasil. \\ ${ }^{4}$ Universidade Federal Rural do Semi-Árido, Mossoró, RN, Brasil. \\ *Autora para contato - lucia.daniel.machado@hotmail.com
}

\section{Resumo:}

Este estudo foi realizado para determinar o efeito de hidroxitolueno butilado (BHT) sobre a qualidade do sêmen canino congelado e descongelado, utilizando o diluidor à base de água de coco em pó (ACP106c). Para tanto, foram realizadas quinze coletas de sêmen provenientes de cinco cães. $O$ sêmen obtido foi diluído em ACP-106c acrescido de glicerol e gema de ovo. As amostras foram então transferidas para tubos contendo diferentes concentrações de BHT $(0 ; 0,5 ; 1,0$ e 2,0 mM). Em seguida, as amostras foram envasadas, congeladas e armazenadas em nitrogênio líquido. O sêmen coletado foi avaliado in natura quanto aos seguintes parâmetros: coloração, volume da fração espermática, motilidade total, vigor, concentração, morfologia e funcionalidade de membrana espermática. Após uma semana, as amostras foram descongeladas e avaliadas por meio de análise computadorizada, como também foram realizadas análises da funcionalidade de membrana e da morfologia espermática. A motilidade progressiva no grupo BHT 2,0 mM foi significativamente superior $(\mathrm{P}<0,05)$ do que a do grupo BHT $0 \mathrm{mM}(27,6 \pm 11,7 \%$ vs. 19,0 $\pm 9,5 \%$, respectivamente). Em todos os demais parâmetros avaliados, não houve diferença entre os grupos testados. Portanto, conclui-se que a adição do BHT ao diluidor ACP-106c não afetou a qualidade do sêmen canino pós-descongelação.

Palavras-chaves: antioxidantes; cão; criopreservação; diluidores.

\begin{abstract}
:
This study was conducted to determine the effect of butylated hydroxytoluene (BHT) on the quality of canine sperm frozen and thawed using the powdered coconut water based (ACP-106c) extender. Therefore, fifteen ejaculates were collected from five dogs. Semen obtained was diluted in ACP-106c added of glycerol and egg yolk. The samples were then transferred to tubes containing different concentrations of BHT $(0,0.5,1.0$ and $2.0 \mathrm{mM})$. After that, the samples were filled into straws, frozen and stored in liquid nitrogen. Fresh semen was evaluated for the following parameters: color, sperm fraction volume, total motility, vigor, concentration, morphology, and HOST test. After one week,
\end{abstract}


the samples were thawed and evaluated by computer analysis, as well as for membrane functionality and sperm morphology. Progressive motility in the $2.0 \mathrm{mM}$ BHT group was significantly higher (P $<0.05)$ than that of the $0 \mathrm{mM}$ BHT group $(27.6 \pm 11.7 \%$ vs. $19.0 \pm 9.5 \%$, respectively). Regarding all other parameters evaluated, there was no difference between the groups tested. Therefore, the addition of BHT to the ACP-106c extender did not affect the quality of canine semen after thawing.

Keywords: antioxidants; cryopreservation; dog; extenders.

Recebido em: 14 de março de 2017

Aceito em: 21 de março de 2018

\section{Introdução}

Um dos principais pontos que podem determinar o sucesso na criopreservação de sêmen é a escolha do melhor diluidor ${ }^{(1)}$. Apesar de o diluidor à base de Tris ser reconhecido mundialmente como melhor diluente para sêmen de cães, tem sido proposta a utilização de diluentes alternativos, que forneçam uma excelente qualidade pós-descongelamento, que sejam de baixo custo e fácil preparo. Dentre esses, surge a água de coco em pó (ACP-106c) $)^{(2)}$, a qual já foi utilizada na diluição do sêmen de cães para recuperação de espermatozoides epididimários ${ }^{(3)}$, fertilização in vitro ${ }^{(4)}$ e inseminação $\operatorname{artificial}^{(5)}$, obtendo bons resultados. Entretanto, em relação à criopreservação de sêmen, independente do diluidor utilizado, sabe-se que ocorre uma significativa redução nos parâmetros espermáticos gerais ${ }^{(6)}$, logo é necessária uma melhoria nos protocolos atuais de congelação.

$\mathrm{Na}$ criopreservação, é certo que ocorre um aumento na quantidade de espécies reativas de oxigênio (ERO), quando estes são capazes de danificar componentes celulares como DNA, lipídios e proteínas $^{(7,8)}$. O estresse oxidativo durante esse processo é a causa principal de morte celular e da baixa fertilidade após a inseminação artificial com sêmen descongelado ${ }^{(9)}$. A geração de EROs é uma consequência normal do metabolismo oxidativo e está ligada diretamente a danos causados aos espermatozoides $^{(10)}$. A suscetibilidade particular da membrana espermática ao dano oxidativo se deve a um elevado conteúdo celular de ácidos graxos poli-insaturados e a uma deficiência de enzimas protetoras nos espermatozoides, visto que eles perdem a maior parte de seu citoplasma durante espermiogênese ${ }^{(11)}$.

Uma maneira de superar os efeitos prejudiciais das EROs é a adição de compostos antioxidantes ao diluidor, para bloquear ou prevenir o estresse oxidativo ${ }^{(12)}$. Existem muitas variedades de antioxidantes que podem ser utilizados, dentre eles o hidroxitolueno butilado (BHT). O efeito do BHT sobre a qualidade do sêmen já foi testado, obtendo-se excelentes resultados pós-descongelação em varrões $^{(13)}$, perus ${ }^{(12)}$, búfalos ${ }^{(14)}$, touros ${ }^{(15)}$, bodes $^{(16,17)}$, carpas $(\text { Cyprinus carpio) })^{(18)}$ e homens ${ }^{(19)}$. Entretanto, nos trabalhos já realizados em cães, é possível verificar resultados contraditórios, no qual Neagu et al. ${ }^{(20)}$ descrevem uma melhoria na qualidade seminal em dois diluidores à base de Tris adicionados de BHT, enquanto no trabalho realizado por Sahashi et al. ${ }^{(21)}$, os resultados não mostraram uma proteção eficiente aos espermatozoides que justificasse sua inclusão ao meio diluidor. Assim, é necessário verificar se a inclusão do BHT a outro diluente tão eficaz quanto o Tris pode contribuir ou não para a melhoria nos parâmetros seminais. Portanto, o objetivo deste trabalho foi verificar o efeito da adição do BHT em diferentes concentrações ao diluidor APC-106c para criopreservação do sêmen canino sobre a qualidade espermática após a descongelação. 


\section{Material e Métodos}

O trabalho foi submetido e aprovado pelo Comitê de Ética para o Uso de Animais da Universidade Estadual do Ceará, registrado sob o número 11516625-4/66.

Previamente, foram selecionados para o experimento apenas aqueles animais que, após a avaliação da qualidade seminal, apresentaram ejaculados com motilidade total igual ou superior a $90 \%$ e vigor igual ou superior a 4. Foram utilizados cinco cães com idade entre dois a seis anos, sendo dois Pastores Alemães, um Retriever Labrador e dois Rottweilers, provenientes do canil da Polícia Militar do Estado do Ceará. Para cada cão, foram realizadas três coletas de sêmen por meio da técnica da manipulação digital com intervalo de uma semana para cada coleta de um mesmo animal. Os cães receberam alimentação à base de ração comercial peletizada super premium e tiveram acesso à água ad libitum.

O sêmen coletado foi avaliado a fresco quanto aos seguintes parâmetros: coloração, aspecto, volume da fração espermática $(\mathrm{mL})$, motilidade total (\%), vigor (0-5), concentração (espermatozoides/mL), morfologia (\% normais) e funcionalidade de membrana espermática $(\%)^{(22)}$. Os parâmetros de motilidade e vigor foram avaliados em uma alíquota de $10 \mu \mathrm{L}$ da amostra de sêmen, através da microscopia óptica, em aumento de 100x. A concentração espermática foi verificada utilizando-se a câmara de Neubauer. Para a avaliação da morfologia foram realizados esfregaços de sêmen, corados com Rosa de Bengala e, posteriormente, avaliados utilizando-se um microscópio óptico (1000x), realizando análise em 200 células espermáticas. Os espermatozoides foram classificados como normais ou apresentando alterações morfológicas de acordo com a sua localização (cabeça, peça intermediária, cauda e acrossoma). Para o teste de funcionalidade de membrana (teste hiposmótico HOST), $10 \mu \mathrm{L}$ de sêmen foi diluído em $90 \mu \mathrm{L}$ de solução hiposmótica $(300 \mathrm{mOsm} / \mathrm{L})$ e mantido em banho-maria a $38{ }^{\circ} \mathrm{C}$. Após 45 minutos, foram avaliadas 100 células em microscopia óptica em aumento de 400x. Os espermatozoides que apresentaram sua cauda enrolada foram considerados com membrana espermática funcional ${ }^{(23)}$.

No laboratório, diferentes concentrações de 3,5-Di-tert-4-butylhydroxytoluene (BHT, SIGMAALDRICH, São Paulo-SP, Brasil), sendo: 0,5 mM; 1,0 mM e 2,0 mM, e uma amostra sem BHT foram preparadas em tubos de ensaio junto com etanol pré-aquecido a $37{ }^{\circ} \mathrm{C}^{(14)}$.

Foi utilizado um diluidor à base de água de coco em pó (ACP-106c, ACP Biotecnologia ${ }^{\circledR}$, FortalezaCeará, Brasil), que foi preparado de acordo com a orientação do fabricante. No local de coleta, o sêmen foi diluído em tubos de vidro já contendo o diluidor ACP-106c, juntamente com $10 \%$ de gema de ovo, $6 \%$ de glicerol, ainda sem o antioxidante. Após essa diluição em temperatura ambiente (aproximadamente $27^{\circ} \mathrm{C}$ ), as amostras foram armazenadas em recipientes com água e acondicionadas em caixa térmica com gelo reciclável $\left(15^{\circ} \mathrm{C}\right)$ por 40 minutos. Em seguida, as amostras foram refrigeradas a $4^{\circ} \mathrm{C}$ por 30 minutos em um refrigerador. Posteriormente, as amostras foram transferidas para tubos também resfriados a $4{ }^{\circ} \mathrm{C}$, contendo as diferentes concentrações de BHT. Foram cronometrados 5 minutos após a diluição visando a-captação do antioxidante pelos espermatozoides. Após esta etapa, o sêmen foi então envasado em palhetas de $0,25 \mathrm{~mL}$, a uma concentração final das amostras de $100 \times 10^{6}$ espermatozoides $/ \mathrm{mL}$, e depois dispostas horizontalmente em rampa de congelação a uma altura de $5 \mathrm{~cm}$ do nível de nitrogênio líquido por cinco minutos e, finalmente, armazenadas em nitrogênio líquido ${ }^{(4)}$. 
Uma semana após a congelação, foi realizada a descongelação em banho-maria a uma temperatura de $37^{\circ} \mathrm{C}$ por 1 minuto. Em seguida, as amostras de sêmen foram transferidas para tubos de vidro presentes no mesmo banho-maria. Feito isso, foram realizados esfregaços de sêmen para a avaliação da morfologia espermática conforme descrito para o sêmen fresco, um novo teste de funcionalidade de membrana e foi empregado o sistema de análise computadorizada do sêmen (CASA). Para a realização do CASA foram retirados $5 \mu \mathrm{L}$ da amostra de sêmen presente no tubo de vidro e colocada em uma Câmara de Makler ${ }^{\circledR}$ (Sel-Medical Instruments, Haifa, Israel), previamente aquecida a $37{ }^{\circ} \mathrm{C}$ e, finalmente, realizada a avaliação ${ }^{(3)}$. As avaliações foram executadas através de um microscópio de contraste de fases acoplado a uma videocâmara adaptada ao sistema Sperm Class Analyser ${ }^{\circledR}$ (SCA, Microptic S.L., versão 5.2.0, Barcelona, Espanha). Três diferentes campos microscópicos, não consecutivos, foram selecionados aleatoriamente por varredura. As configurações do CASA foram: número de imagens adquiridas, (25 por segundo), contraste mínimo da célula 75 ; índice de progressão, 75\%; velocidade mínima (VAP - velocidade média da trajetória), 10, e velocidade média (VAP), 55(24). Os parâmetros mensurados para cada amostra foram: motilidade total (MT) (\%), motilidade progressiva (MP) (\%), velocidade curvilinear (VCL) $(\mu \mathrm{m} / \mathrm{s})$, velocidade progressiva $(\mathrm{VSL})(\mu \mathrm{m} / \mathrm{s})$, velocidade média da trajetória (VAP) $(\mu \mathrm{m} / \mathrm{s})$, índice de linearidade (LIN) (\%), índice de progressão (STR) (\%), amplitude lateral da cabeça (ALH), frequência de batimento cruzado $(\mathrm{BCF})^{(24)}$.

Os dados foram checados quanto à sua normalidade pelo teste de Shapiro-Wilk e quanto à homocedasticidade pelo teste de Levene, usando o software estatístico StatView 5.0. Valores expressos em porcentagem foram transformados em Arcoseno antes da análise. A comparação entre os diferentes tratamentos foi realizada por meio de análise de variância, seguida do teste t de Student. Os dados foram expressos sob a forma de média \pm desvio padrão. Diferenças foram consideradas significativas quando $\mathrm{p}<0,05$.

\section{Resultados}

Em todas as coletas de sêmen, foi utilizada apenas a segunda fração do ejaculado (fração espermática). Não foram verificadas falhas nas coletas, sendo obtido sêmen em todas as tentativas. Em adição, não foram constatadas contaminações com urina ou sangue, sendo todas de coloração branca e aspecto leitoso. Os parâmetros seminais avaliados a fresco encontram-se na tabela 1.

Tabela 1. Parâmetros espermáticos in natura (média \pm DP)

\begin{tabular}{lc}
\hline \multicolumn{1}{c}{ Parâmetros } & Valores \\
\hline Volume (mL) & $1,9 \pm 0,4$ \\
Motilidade total (\%) & $98,7 \pm 2,3$ \\
Vigor (0-5) & $5 \pm 0$ \\
Concentração (espermatozoides $/ \mathrm{mL})$ & $1020,7 \pm 302,4 \times 10^{6}$ \\
Funcionalidade de membrana (\%) & $93,3 \pm 4,3$ \\
Morfologia normal (\%) & $93,1 \pm 4,7$ \\
\hline
\end{tabular}

Quanto à análise do sêmen descongelado, a motilidade progressiva do grupo adicionado de BHT 2,0 $\mathrm{mM}$ foi significativamente superior ao grupo sem BHT $(27,6 \pm 11,7 \%$ vs. $19 \pm 9,5 \%, \mathrm{P}<0,05)$. Os 
grupos adicionados de BHT 0,5 e 1,0 mM não diferiram dos demais. Os demais parâmetros avaliados pela CASA, bem como a funcionalidade de membrana espermática não foram afetados pela adição de BHT. A percentagem de espermatozoides morfologicamente normais reduziu significativamente $(\mathrm{P}<0,05)$ em todos os grupos comparados à amostra in natura. No entanto, a concentração de BHT não influenciou a morfologia espermática após a descongelação $(\mathrm{P}<0,05$ - Tabela 2$)$.

Tabela 2. Valores (média \pm DP) da CASA e de espermatozoides com membrana funcional para o sêmen canino descongelado diluído em ACP-106C e acrescido ou não de BHT

\begin{tabular}{|c|c|c|c|c|}
\hline \multirow[t]{2}{*}{ Parâmetros espermáticos } & \multicolumn{4}{|c|}{ Concentrações de BHT } \\
\hline & 0 & 0,5 & 1,0 & 2,0 \\
\hline MT (\%) & $53,7 \pm 25,2^{a}$ & $68,2 \pm 17,4^{a}$ & $63,0 \pm 20,1^{a}$ & $69,0 \pm 19,2^{a}$ \\
\hline MP (\%) & $19,0 \pm 9,5^{b}$ & $21,5 \pm 12,8^{a b}$ & $27,8 \pm 15,3^{a b}$ & $27,6 \pm 11,7^{\mathrm{a}}$ \\
\hline $\mathrm{VCL}(\mu \mathrm{m} / \mathrm{s})$ & $70,7 \pm 17,6^{a}$ & $60,7 \pm 15^{a}$ & $71,6 \pm 16,3^{a}$ & $68,3 \pm 17,4^{a}$ \\
\hline $\mathrm{VSL}(\mu \mathrm{m} / \mathrm{s})$ & $40,0 \pm 17,3^{a}$ & $34,5 \pm 15,1^{a}$ & $41,5 \pm 14,4^{a}$ & $38,7 \pm 20,2^{a}$ \\
\hline $\operatorname{VAP}(\mu \mathrm{m} / \mathrm{s})$ & $49,9 \pm 15,5^{a}$ & $44,5 \pm 15,1^{a}$ & $51,6 \pm 14,2^{a}$ & $49,5 \pm 18,4^{a}$ \\
\hline LIN (\%) & $54,8 \pm 14,1^{a}$ & $54,7 \pm 12,6^{a}$ & $56,8 \pm 12,4^{a}$ & $54,9 \pm 16,3^{a}$ \\
\hline STR (\%) & $77,4 \pm 10,7^{a}$ & $75,2 \pm 9,1^{\mathbf{a}}$ & $78,8 \pm 9,5^{\mathrm{a}}$ & $75,4 \pm 13,4^{\mathrm{a}}$ \\
\hline $\mathrm{ALH}(\mu \mathrm{m})$ & $3,6 \pm 0,9^{a}$ & $3,3 \pm 0,5^{\mathbf{a}}$ & $3,4 \pm 0,6^{a}$ & $3,3 \pm 0,6^{a}$ \\
\hline $\mathrm{BCF}(\mathrm{Hz})$ & $9,5 \pm 2,1^{\mathrm{a}}$ & $9,6 \pm 2,9^{a}$ & $10,1 \pm 2,6^{a}$ & $9,5 \pm 2,6^{\mathbf{a}}$ \\
\hline Membrana funcional (\%) & $73,0 \pm 11,6^{\mathrm{a}}$ & $72,6 \pm 7,9^{a}$ & $74,3 \pm 7,2^{\mathbf{a}}$ & $79,2 \pm 5,7^{\mathrm{a}}$ \\
\hline Morfologia normal (\%) & $77,6 \pm 7,2^{\mathrm{a}}$ & $74,9 \pm 8,2^{\mathrm{a}}$ & $78,9 \pm 8,1^{\mathrm{a}}$ & $74,3 \pm 7,9^{a}$ \\
\hline
\end{tabular}

Letras minúsculas diferentes representam diferença estatística entre colunas $(\mathrm{P}<0,05)$.

Motilidade total (TM), motilidade progressiva (PM), velocidade curvilinear (VCL), velocidade progressiva (VSL), velocidade média da trajetória (VAP), indice de linearidade (LIN), indice de progressão (STR=VSL/VAP), amplitude lateral de cabeça (ALH), frequência de batimento cruzado (BCF), funcionalidade de membrana e morfologia normal.

\section{Discussão}

Os valores encontrados inicialmente para os parâmetros de motilidade total, vigor, concentração, morfologia normal e funcionalidade da membrana do sêmen fresco, refletem uma excelente qualidade espermática encontrada nestes machos. Tais fatores são um bom indicativo de fertilidade, como também é possível afirmar que as amostras se encontram aptas à congelação ${ }^{(25)}$.

Em relação aos valores encontrados após a descongelação, foi possível perceber uma melhor qualidade da motilidade progressiva dos espermatozoides na concentração de 2,0 $\mathrm{mM}$ de BHT (27,6\%), quando comparado ao grupo sem o antioxidante $(19,0 \%)$. Esse resultado difere do encontrado por Ghorbani et al. ${ }^{(19)}$ em humanos, pois relataram que concentrações de BHT superiores a $0,5 \mathrm{mM}$ resultaram em menor motilidade e viabilidade espermática provavelmente devido a um aumento de ânions superóxido e $\mathrm{H}_{2} \mathrm{O}_{2}$ nos espermatozoides. Khumran et al. ${ }^{(26)}$ relataram uma toxicidade causada pelo BHT, quando este é utilizado em concentrações maiores de $1 \mathrm{mM}$ em sêmen de touros, levando a redução da motilidade e da viabilidade espermática. O mecanismo relacionado à toxicidade deste antioxidante ainda não se encontra totalmente elucidado, mas, ao que parece, o espermatozoide do cão parece ser mais resistente a este efeito deletério.

Nos demais parâmetros espermáticos avaliados pelo software SCA ${ }^{\circledR}$, não foram verificadas 
diferenças entre os grupos avaliados. Resultado similar foi descrito por Neagu et al. ${ }^{(20)}$, que avaliaram a ação do antioxidante sobre o sêmen canino diluído em dois diferentes diluidores, o Uppsala e o INRA-96, sendo também verificado que a adição do BHT não interfere nos parâmetros das motilidades total e linear e das velocidades linear, curvilinear e média.

Com a adição do BHT, esperava-se que houvesse uma maior proteção à membrana espermática ${ }^{(20,27)}$. Por ser solúvel em lipídios, o BHT tem a propriedade adicional de aumentar a fluidez das membranas espermáticas $^{(28)}$, melhorando assim a viabilidade dos espermatozoides através da proteção à membrana celular. Entretanto, não foi verificada uma proteção efetiva, pois os resultados das amostras com BHT não foram superiores ao grupo controle na análise de funcionalidade de membrana. Esse resultado demonstrou uma ineficiência do antioxidante em um dos principais sítios onde sua proteção deveria ser efetiva, pois a membrana plasmática dos espermatozoides é o principal local onde os EROs interagem devido à presença de uma maior quantidade de ácidos graxos poliinsaturados ${ }^{(29,30)}$.

Com exceção da motilidade espermática, nos demais parâmetros avaliados, as amostras adicionadas de BHT em nenhum momento obtiveram resultados superiores ao grupo controle, independente da concentração utilizada. O mecanismo de ação de BHT em células espermáticas é principalmente através de uma proteção dos lipídios da membrana, contra uma desnaturação química feita pelos EROs. As células podem, portanto, manter a morfologia básica e seu estado físiológico como um resultado das ações de proteção efetuadas pelo $\mathrm{BHT}^{(26)}$.

Os resultados observados para a concentração de $0,5 \mathrm{mM}$ não diferiram dos resultados encontrados sem a inclusão do BHT ao sêmen diluído. Da mesma forma foi descrito por Anderson et al. ${ }^{(31)}$, em que a adição de $0,5 \mathrm{mM}$ de BHT ao diluidor seminal antes da congelação não melhorou a taxa de fertilidade em touros. Outros trabalhos com bovinos também relataram que a adição do BHT não melhora a qualidade seminal ${ }^{(32)}$.

No estudo realizado por Sahashi et al. ${ }^{(21)}$, que também avaliaram o sêmen canino descongelado, foi verificado que não há diferença significativa entre as amostras sem o BHT das amostras com BHT até a concentração de $0,8 \mathrm{mM}$. A redução na qualidade espermática foi verificada apenas na concentração mais alta $(1,6 \mathrm{mM})$, assim, diferenciando de nosso trabalho no qual a concentração de 2,0 mM foi a mais alta utilizada e a única que apresentou melhor resultado que o grupo sem BHT em relação à motilidade progressiva, mas não diferindo nos demais parâmetros avaliados. Provavelmente, isso denota uma interação bioquímica diferenciada entre o BHT e os componentes do diluente ACP-106c, o que deve então favorecer a motilidade dos espermatozoides caninos.

O efeito da proteção de danos conferida aos espermatozoides pelo BHT depende da espécie, da concentração de BHT, da composição da membrana celular, do tempo de incubação e, principalmente, do tipo de diluidor ${ }^{(1)}$. Entretanto, dos trabalhos realizados na espécie canina, os diluidores até então testados eram à base de Tris e, mesmo assim, foram obtidos resultados contraditórios.

\section{Conclusão}

No presente estudo, a inclusão de BHT ao diluidor ACP-106c obteve resultado superior ao grupo controle somente em relação à motilidade progressiva, enquanto, nos demais parâmetros, foi 
indiferente, assim não havendo justificativa para sua adição ao meio diluidor. Logo, conclui-se que a adição do BHT ao diluidor ACP-106c não afetou a qualidade do sêmen canino pós-descongelação.

\section{Referências}

1. Roca J, Gil MA, Hernández M, Parrilla I, Vazquez JM Martinez EA. Survival and fertility of boar spermatozoa after freeze thawing in extender supplemented with butylated hydroxytoluene. Journal of Andrology. 2004; 25(3): 397-405.

2. Cardoso RCS, Silva AR, Silva LDM. Use of the powdered coconut water (ACP 106) for cryopreservation of canine spermatozoa. Animal Reproduction. 2005; 2(4): 257-262.

3. Mota Filho AC, Silva HVR, Nunes TGP, Souza MB, Freitas LA, Araújo AA, Silva LDM. Cryopreservation of canine epididymal sperm using ACP-106c and TRIS. Cryobiology. 2014; 69(1) 17-21.

4. Cardoso RCS, Silva AR, Silva LDM., Chirinéa VH, Souza FF, Lopes MD. Evaluation of fertilizing potential of frozen-thawed dog spermatozoa diluted in ACP-106 ${ }^{\circledR}$ using an in vitro sperm-oocyte interaction assay. Reproduction in Domestic Animals. 2007; 42(1):11-16.

5. Uchoa DC, Silva TFP, Cardoso JFS, Mota Filho AC, Jucá RP, Silva AR, Silva LDM. Favoring the birth of female puppies after artificial insemination using chilled semen diluted with powdered coconut water (ACP106c). Theriogenology. 2012; 77(9):1959-1963.

6. Silva AR, Fontenele-Neto JD, Cardoso RCS, Silva LDM, Chirinéa VH, Lopes MD. Description of ultrastructural damages in frozen-thawed canine spermatozoa. Ciência Animal Brasileira. 2009; 10(2):595501.

7. Thomson LK, Fleming SD, Aitken RJ, Iuliis GN, Zieschang JA, Clark AM. Cryopreservation-induced human sperm DNA damage is predominantly mediated by oxidative stress rather than apoptosis. Human Reproduction. 2009; 24(9): 2061-2070.

8. Zribi N, Chakroun NF, Ben Abdallah F, Elleuch H, Sellami A, Gargouri J, Rebai T, Fakhfakh F, Keskes LA. Effect of freezing-thawing process and quercetin on human sperm survival and DNA integrity. Cryobiology. 2012; 65(3): 326-331.

9. Seifi-Jamadi A, Kohrama H, Zareh-Shahne A, Dehghanizadeh P, Ahmad E. Effect of various concentrations of butylated hydroxyanisole and butylated hydroxytoluene on freezing capacity of Turkman stallion sperm. Animal Reproduction Science. 2016; 170: 108-113.

10. Maxwell WM, Stojanov T. Liquid storage of ram semen in the absence or presence of some antioxidants. Reproduction Fertility and Development. 1996; 8(6):1013-1020.

11. Halliwell B, Gutteridge JMC. Free Radicals in Biology and Medicine. $4^{\text {th }}$ ed. Oxford University Press; 2007. 704p.

12. Donoghue AN, Donoghue DJ. Effects of water and lipid-soluble antioxidants on turkey sperm viability, membrane integrity, and motility during liquid storage. Poultry Science. 1997; 76(10): 1440-1445.

13. Trzcińska M, Bryła M, Gajda B, Gogol P. Fertility of boar semen cryopreserved in extender supplemented with butylated hydroxytoluene. Theriogenology. 2015; 83(3): 307-313.

14. Ijaz A, Hussain A, Aleem M, Yousaf MS, Rehman H. Butylated hydroxytoluene inclusion in semen extender improves the post-thawed semen quality of Nili-Ravi buffalo (Bubalus bubalis). Theriogenology. 2009; 71(8):1326-1329. 
15. Shoae A, Zamiri MJ. Effect of butylated hydroxytoluene on bull spermatozoa frozen in egg yolk-citrate extender. Animal Reproduction Science. 2008; 104(2-4): 414-418.

16. Khalifa TA, Lymberopoulos AG, El-Saidy BE. Testing usability of butylated hydroxytoluene in conservation of goat semen. Reproduction in Domestic Animal. 2008; 43(5): 525-530.

17. Naijian HR, Kohram H, Shahneh AZ, Sharafi M, Bucak M. Effects of different concentrations of BHT on microscopic and oxidative parameters of Mahabadi goat semen following the freeze-thaw process. Cryobiology. 2013; 66(2): 151-155.

18. Ogretmen F, İnanan BE. Effect of butylated hydroxytoluene (BHT) on the cryopreservation of common carp (Cyprinus carpio) spermatozoa. Animal Reproduction Science. 2014; 151(3-4): 269-274.

19. Ghorbani M, Amiri I, Khodadadi I, Fattahi A, Atabakhsh M, Tavilani H. Influence of BHT inclusion on post-thaw attributes of human semen. Systems Biology in Reproductive Mededicine. 2014; 61(1): 57-61.

20. Neagu VR, García BM, Sandoval CS, Rodríguez AM, Ferrusola CO, Fernández LG, Tapia JÁ, Peña FJ. Freezing dog semen in presence of the antioxidant butylated hydroxytoluene improves postthaw sperm membrane integrity. Theriogenology. 2010; 73(5): 645-650.

21. Sahashi Y, Otsuki T, Higaki S, Nagano M, Yamashita Y, Hishinuma M. Effect of butylated hydroxytoluene on dog sperm longevity in chilling storage and cryopreservation. The Journal of Veterinary Medical Science. 2011; 73(7): 895-899.

22. Cardoso RCS, Silva AR, Silva LDM. Métodos de avaliação do sêmen canino congelado. Revista Brasileira de Reprodução Animal. 2005; 29(3-4):179-187. Português.

23. Quintela AT, Oliveira IRS, Souza AO, Gusmão AL, Silva AR. Water-induced hypo-osmotic test for the evaluation of canine sperm membrane integrity. Animal Reproduction. 2010; 7(2): 70-74.

24. Rijsselaere T, Maes D, Hoflack G, Kruif A, Soom AV. Effect of body weight, age and breeding history on canine sperm quality parameters measured by the Hamilton-Thorne Analyser. Reproduction in Domestic Animals. 2007; 42(2): 143-148.

25. Robert MA, Jayaprakash G, Pawshe M, Tamilmani T, Sathiyabarathi M. Collection and evaluation of canine semen-a review. International Journal of Science. 2016; 5(3):1586-1595.

26. Khumran AM, Yimer N, Rosnina Y, Ariff MO, Wahida H, Kaka A, Ebrahimi M, Sarsaifi K. Butylated hydroxytoluene can reduce oxidative stress and improve quality of frozen-thawed bull semen processed in lecithin and egg yolk based extenders. Animal Reproduction Science. 2015; 163:128-134.

27. Graham JK, Hammerstedt R. Differential effects of butylated hydroxytoluene analogs in bull sperm subjected to cold induced membrane stress. Cryobiology. 1992; 29(1):106-17.

28. Asadpour R, Jafari R, Tayefi-Nasrabadi H. The effect of antioxidant supplementation in semen extenders on semen quality and lipid peroxidation of chilled bull spermatozoa. Iranian Journal of Veterinary Research. 2012; 13(3): 246-249.

29. Aitken RJ, Jones KT, Robertson SA. Reactive oxygen species and sperm function in sickness and in health. Journal of Andrology. 2012; 33(6):1096-1106.

30. Alvarenga MA, Papa FO, Carmo MT, Kievitsbosch T, Castro Chaves MMB, Ramires Neto C. Methods of concentrating stallion semen. Journal of Equine Veteterinary Science. 2012; 32(8): 424-429.

31. Anderson S, Harkness W, Akin Y, Kaproth M, Killian G. Categorical data analysis of the effect on bull fertility of butylated hydroxytoluene addition to semen extenders prior to freezing. J. Dairy Sci. 1994; 77(8):2302-2307. 
32. Suttiyotin P, Nakthong C, Kimsakulvech S, Jarearnkul D. Effect of butylated hydroxytoluene on survival of frozen-thawed fighting bull spermatozoa. Journal of Agricultural Science and Technology. 2011; 1:11771180. 